\title{
AMENORREA - Ponencia oficial
}

\author{
SOCIEDAD VALLECAUCANA DE OBSTETRICIA Y GINECOLOGIA \\ $X$ CONGRESO NACIONAL DE OBSTETRICIA Y GINECOLOGIA \\ Presentado por los Dres.:
}

\author{
Fernando del Corral G. 梚 \\ Jorge E. Zúñiga C. 深 \\ Con la colaboración de: \\ Jairo de la Cruz祭将

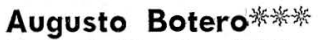

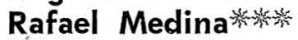 \\ Hugo Villegas燃棌棌
}

Y los Departamentos de:
PATOLOGIA Y RADIOLOGIA - Facultad de Medicina - Universidad del Valle - Cali, Colombia S. A.

\section{Introducción}

El presente estudio comprende el análisis de 148 casos de pacientes que consultaron o fueron remitidos a la consulta especializada de Ginecología Endocrina del Hospital Universitario del Valle Departamento de Obstetricia y Ginecología Facultad de Medicina Universidad del Valle.

El análisis comprende 10 años de estudio desde Julio de 1963 a Julio de 1973.

Como esta entidad es uno de los problemas de más difícil diagnóstico y manejo en Ginecología, los casos siempre fueron analizados y manejados por un mismo grupo de observadores para conservar la misma unidad de criterio siguiendo la clasificación de León Israel en su texto "Trastornos Menstruales y Esterilidad" (18) Cuadro N: 1 CON LA ADICION DE la
Amenorrea Central Idiopática y la Menopausia Precoz, los cuales no figuran en la misma.

En esta clasificación se ha entendido como Amenorrea la suspensión de la menstruación por 90 o más días o cuando ésta no se hubiere presentado a la edad máxima de 18 años.

En algunas oportunidades las causas de la Amenorrea fueron múltiples, casos en los cuales las pacientes se clasificaron de acuerdo al factor ETIOLOGICO DOMINANTE. La orientación de la paciente, para el estudio analítico correspondiente de su problema, se basó inicialmente en los hallazgos del examen clínico, el cual nos orientó la mayoría de los casos, para orde-

* Profesor Dpto. de Obstetricia y Ginecología.

* Auxiliar de Cátedra. Dpto. de Morfología.

*** Residentes Hospital Universitario "Evaristo García". 
nar los exámenes pertinentes comprobatorios, tratando de evitar pérdida de tiempo y gastos innecesarios a la paciente.

En esta clínica contamos con los siguientes procedimientos de laboratorio:

\section{Hipotálamo - Hipófisis}

a) Determinación Gonadotropinas Urinarias Totales, por el método de concentración de Bradbury (7) y Bioensayo de Clinefelter (22).

b) Test de Reserva Pituitaria con SU 4885 (Metopirona) el cual se realizó en pacientes.

c) Estudios Radiológicos.

\section{Tiroides}

PBI - Yodo 131 - Estudios de T3 T4 Gomagrafía.

\section{Adrenales}

Test de estimulación con ACTH y supresión con dexametazona interpretados en orina y sangre.

Determinación de 17 oxosteroides y 17 hidroxicorticoides totales.

Sólo recientemente se han incorporado los métodos de Dosificación de Estrógenos, Progesterona y Andrógenos.

\section{Gónadas}

Citología funcional.

Biopsia de endometrio.

(Microscopia de fase y Microscopia electrónica).

Test de estimulación ovárica con Gonadotropina Coriónica, previo freno con Dexametazona.

Test de Progesterona.

Biopsia de ovario por culdoscopia (análisis histológico topográfico del ovario).

\section{Otros}

Cromatina sexual y cariotipo. Exámenes radiológicos correspondientes.

Exámenes especiales para tuberculosis, toxoplasmosis, diabetes etc.

\section{Resultados}

No podemos presentar la verdadera incidencia de "amenorreaí" en nuestro medio debido a la falta de selección del grupo estudiado.

En los diferentes grupos de pacientes que a continuación se presentan según clasificación ya mencionada, se hará un análisis del manejo y resultado en los grupos más representativos.

Estos resultados se muestran en el Cuadro No 3.

\section{SISTEMA NERVIOSO CENTRAL (HIPOTALAMICO)}

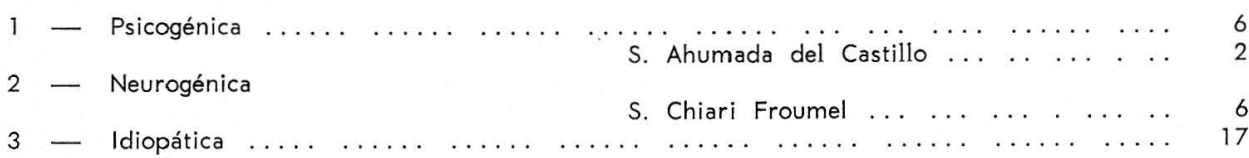

\section{GLANDULA PITUITARIA}

1 - Hipofunción con obesidad $\ldots \ldots \ldots \ldots \ldots \ldots \ldots \ldots \ldots \ldots$

2 - Necrosis hipofisiaria (Sheehan) $\ldots \ldots \ldots \ldots \ldots \ldots \ldots \ldots$

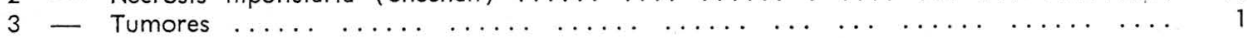




\section{GONADAS (OVARIOS)}

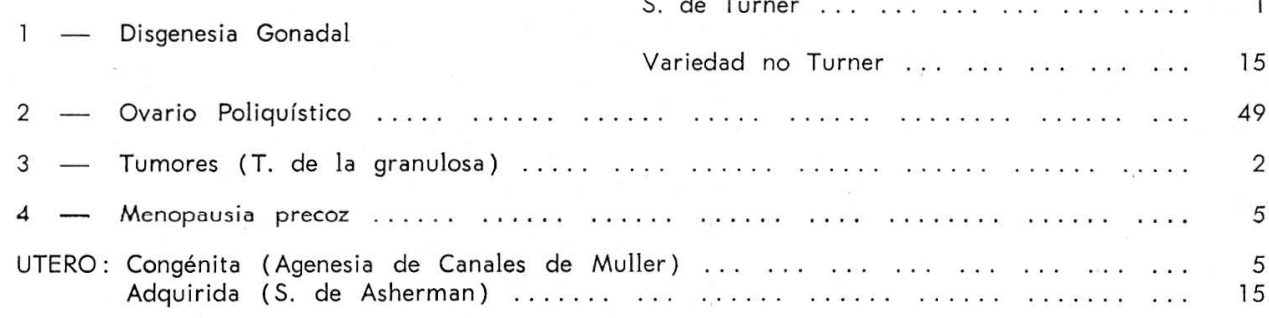

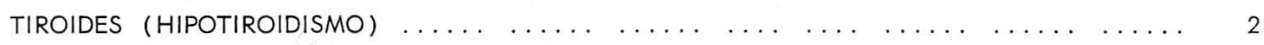

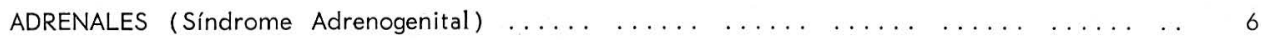

NUTRITIVA 0 METABOLICA $\ldots \ldots \ldots$

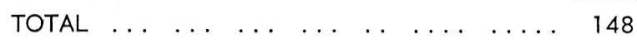

De acuerdo con lo expuesto en la Tabla No 1, la causa de Amenorrea más frecuente se encontró en el grupo de Ovario Poliquístico y/o Síndrome de Stein-Leventhal. En este Síndrome se incluyeron como Stein-Leventhal aquellas pacientes con: 1 alteraciones del ciclo; 2 esterilidad; 3 ovarios poliquísticos; 4 obesidad $\mathrm{y} / \mathrm{o}$ hirsutismo. Todas las pacientes estudiadas tuvieron su examen clínico general completo, y la mayoría, los exámenes de rutina pertinentes, incluyendo citología funcional, biopsia de endometrio, 17 oxosteroides, 17 hidroxicorticoides, estudios planimétricos del área del ovario de acuerdo a lo expuesto por Weigen (37) y en algunos casos pruebas de estimulación con ACTH, supresión con Dexametasona y estimulación con Gonadotropinas Coriónicas. Cuando se creyó necesario, se realizó la culdoscopia.

Los estudios histológicos del ovario se hicieron con el mismo grupo de observadores y con los mismos parámetros, realizándose coloraciones especiales cuando éstas estuvieron indicadas.
Creemos que haciendo una revisión de los casos de Stein-Leventhal como grupo, nos daremos una mejor idea de esta patología entre nosotros; es por esto que hemos incluído todos los casos con alteraciones del ciclo. Se revisaron un total de 95 casos durante la década del 63 al 73, de los cuales llenaron los requisitos para estudio y análisis: 69 casos. La mayoría 49 pacientes $(52 \%)$ estaban en Amenorrea, 22 con Oligomenorrea $(23 \%), 6$ con Polimenorrea (6\%), 1 caso ( $1 \%$ ) con Metrorragia y 17 casos sin dato en la historia clínica $(17 \%)$.

Los datos clínicos más sobresalientes fueron además de las alteraciones ciclo: Obesidad 57 pacientes $(60 \%)$, Hirsutismo 53 pacientes $(56,7 \%)$, Ovarios clínicamente grandes 17 pacientes $(6 \%)$, Ovarios radiológicamente grandes (Ginecografías) en 53 casos $(57 \%)$.

\section{Edad de las pacientes}

Osciló6 entre los 16 y 45 años, con la siguiente distribución por grupos: Tabla № 2. 
De 15 a 19 años: 16 casos

De 20 a 24 años: 41 "

De 25 a 29 años: 20 "

De 30 a 34 años: 11 ',

De 35 a 39 años: 6 "

De más de 40 años : 1 " TOTAL :

Llama la atención que el grupo más significativo se encuentra entre los 20 a 24 años.

\section{Tratamiento}

De las 95 pacientes, se llevaron a cirugía (Resección cuneiforme bilateral), sólo 53 de ellas (57\%), debido a que 19 pacientes $(20 \%)$ de las 95, eran solteras $\mathrm{y} / 0$ de muy temprana edad. 17 pacientes (18\%) se manejaron médicamente, 9 con prednisona, 6 con Terapia Cíclica con anovuladores y 4 con citrato de clomifeno; las 4 pacientes restantes no regresaron a control.

De las 53 pacientes intervenidas, sólo tuvieron controles post-operatorios adecuados, 44 ( $83 \%)$ y 9 no regresaron.

De este grupo intervenido, se encontró que tenían actividad sexual 44 pacientes $(83 \%)$ de las cuales se obtuvo embarazo en 13 pacientes (30\%). De ellas cuatro obtuvieron fetos viables normales, 3 por parto normal y 1 por cesárea por desproporción fetopélvica; 4 terminaron en aborto; 2 de ellas a repetición y en 5 pacientes con embarazo comprobado, no se obtuvo el resultado final de los productos.

Del total de pacientes manejadas médicamente no hay información sobre embarazos.

\section{Estudios de Laboratorio}

Como es de suponer la mayor parte de las pacientes tuvieron una citología con índices de conificación promedio de $86 \%$.
Los 17 exosteroides oscilaron entre 1,8 mgs./24 horas y 14,9 mgs./24 horas, con un valor promedio de 6,09 mgs./24 horas. Esto demuestra de nuevo que los valores totales de 17 exosteroides se encuentran dentro de límites normales en el Síndrome de Ovario Poliquístico y que sólo estudios especiales de fraccionamiento pudieron tener un valor en el diagnóstico diferencial del Síndrome. Por la experiencia con el manejo de pacientes con Síndromes de Hirsutismo - Hipertricosis y en general con cuadros de androgenismo, hemos observado que los 17 exosteroides con cifras superiores a $10 \mathrm{mgs} . / 24$ horas son muy escasos y por lo tanto, las cifras promedio (5 a 15 mgs.) publicadas en la literatura como "normales" son muy altas para nosotros.

En la misma forma las pruebas de estimulación con ACTH, supresión con dexametasona y estimulación con gonadotropina coriónica de acuerdo a lo expuesto por Cooke (9), que podrían tener valor en el diagnóstico diferencial con el Síndrome Adrenogenital, fue realizado en 12 pacientes, los resultados fueron impredecibles y creemos que la prueba en las actuales circunstancias, no sea tan importante para el diagnóstico y manejo de este Síndrome y sí, por el contrario se debe reservar para la orientación diagnóstica y manejo de la mujer hirsuta.

\section{NEUMOPELVIGRAFIA EN SINDROME DE STEIN-LEVENTHAL}

\section{Introducción}

La visualización de las estructuras pélvicas femeninas mediante sistema de contraste con gas intraperitoneal, fue inicialmente descrita por Goetze en 1918 (14).

Estas técnicas no tuvieron mayor difusión debido a problemas derivados del embolismo gaseoso. 
Posteriormente, con la depuración de la técnica y el empleo de gases absorbibles y de rápida y fácil difusión, comenzó a ser reutilizada en los centros clínicos del Instituto Nacional de Salud de los EE. UU. en 1935 para el diagnóstico del Síndrome de Stein-Leventhal (34).

Posteriormente estas técnicas fueron empleadas con alguna frecuencia, en Europa y América Latina y sólo hasta hace muy poco tiempo incorporadas de nuevo en las clínicas radiológicas norteamericanas.

La técnica radiológica propiamente dicha se realiza de acuerdo a los procedimientos clásicos como los descritos por Schultz (33) de los cuales no haremos mención en este escrito por considerarla conocida por ustedes.

De acuerdo a estas técnicas se ha demostrado que este es un método simple que ofrece una buena visualización pélvica de contraste con una morbilidad mínima usando gases de fácil difusión (óxido nitroso y anhídrido carbónico).

No obstante el procedimiento se presenta en problemas de interpretación y es por ello que se han ideado una serie de sistemas más exactos de medición como los descritos por Weigen en 1967 (37), seguido por nosotros, calculando el área del ovario en forma bidimensional. O sistemas más sofisticados como el de Abrhams y colaboradores el cual considera el ovario como una elipse, calculando el área por un sistema tridimensional (1).

El procedimiento por nosotros usado consiste en la técnica originalmente descrita por Stein y Leventhal (37) utilizando $\mathrm{CO}_{2}$ en el lugar del óxido nitroso.

Las lecturas para calcular el área del ovario se hacen multiplicando la longitud por el ancho, expresándose el producto en centímetros cuadrados.

De acuerdo a Weigen, las medidas para el ovario varían desde 3.7 a 14.6 cms.2 con un promedio de $9.0 \mathrm{cms} .2$.

Para el ovario poliquístico, las medidas varían de $6.0 \mathrm{cms} .2$ a $38.0 \mathrm{cms} .2$ con un promedio de $16.8 \mathrm{cms} .2$. Además, una relación ovario-útero de 0.5 - más es sugestivo de enfermedad poliquística de los ovarios. El diagnóstico radiográfico de la enfermedad no puede hacerse solamente mediante el uso de índices, relaciones o valores arbitrarios.

Si se usa un índice de $15 \mathrm{cms}^{2}$ como el tope límite de lo normal, 30\% de los ovarios poliquísticos caerían de límites normales. Si se usa $0.85 \mathrm{co}$ mo el límite superior de la relación normal ovario-útero, $70 \%$ de los ovarios poliquísticos tendrían relaciones dentro de límites normales. Por otro lado, la apariencia típica de los ovarios, simétricamente agrandados, sugiere fuertemente síndrome de ovario poliquístico (Weigen) (37).

\section{PATOLOGIA DEL SINDROME DE STEIN-LEVENTHAL}

Este síndrome no presenta una identificación histológica propia, sino que existe una imagen que le es peculiar para formar parte del mismo. Las alteraciones histológicas se caracterizan por una albugínea engrosada y fibrótica, en ocasiones hialinizada, debajo de la cual se encuentran folículos en diferentes etapas de maduración y atresia, al parecer incapaces de superficializarse para atravesar la corteza| Frecuentemente hay hiperplasia de la teca interna (Hipertecosis).

A esto se le agrega un gran número de folículos quísticos, subcorticales que le dan la apariencia abullonada característica. 
Características histológicas de I Stein-Leventhal.
A. Hipertecosis.
B. Poliquistosis subcortical.
C. Engrosamiento de la albugínea.
D. Ausencia del cuerpo lúteo recien- te o cicatricial.

Inicialmente se consideró que en el cuadro histológico debería haber ausencia de cuerpo amarillo, pero ocasionalmente puede haber una ovulación de escape de manera tal que el hallazgo ocasional de un cuerpo lúteo, no necesariamente excluye la compatibilidad histológica con el síndrome de Stein-Leventhal.

Con el objeto de diferenciar el ovario típico de Stein de aquel con cuerpo lúteo, Shippel, lo incluye dentro del síndrome que lleva su nombre, en aquellos casos en que además de ovulación se asocia el cuadro histológico con una gran hipertecosis (32).

A pesar de estos cuadros histológicos, existen otros que igualmente se asocian con esterilidad, hirsutismo y amenorrea, cuadros éstos de androgenismo, producidos por estromatosis generalizada del ovario o hipertecosis.

Considerando estas variantes histológicas, se revisaron las cuñas de 45 pacientes las cuales se expresan en el cuadro siguiente:

\begin{tabular}{|c|c|c|}
\hline Diagnóstico & No Casos & Porcentaje \\
\hline $\begin{array}{l}\text { Ovario normal } \\
\text { Estromatosis } \mathrm{y} / \mathrm{o}\end{array}$ & 14 & $32 \%$ \\
\hline hipertecosis & 7 & $17 \%$ \\
\hline $\begin{array}{l}\text { Stein-Leventhal } \\
\text { Stein-Leventhal }\end{array}$ & 12 & $25.5 \%$ \\
\hline + cuerpo lúteo & 12 & $25.5 \%$ \\
\hline TOTAL & 45 & $100 \%$ \\
\hline
\end{tabular}

Debido a estos hallazgos pensamos que es probable que el ovario SteinLeventhal y el ovario descrito por
Shippel, con ovulación, son diferentes etapas de evolución de un mismo síndrome.

\section{Resultados}

Se realizaron 23 ginecografías, de las cuales sólo 15 se pudieron estudiar planimétricamente. En las 8 restante sse encontraron dificultades de orden técnico para las mediciones.

De acuerdo a los resultados obtenidos, se encontró un área promedio para el ovario derecho de $14.8 \mathrm{cms}^{2}$ y $18.0 \mathrm{cms}^{2}$ para el ovario izquierdo, con un promedio de 16.4 cms. $^{2}$ para el total de las mediciones. Se obtuvo, además, un área promedio para el útero de $32.3 \mathrm{cms}^{2}$ lo cual dá una relación ovario-útero de 0.5 .

\section{Comentarios}

De acuerdo a los resultados obtenidos, los valores encontrados en nuestro estudio para el Síndrome de ovario poliquístico, coinciden con los revisados en la literatura, considerando al ovario en dos dimensiones.

Con el fin de realizar mediciones lo más exactas posibles, recomendamos la medida del ovario en sus tres dimensiones, aplicando la fórmula matemática de la elipse, para obtener un resultado volumétrico que estaría de acuerdo con la estructura anatómica del ovario.

\section{AMENORREAS CENTRALES ( HIPOTALAMICAS)}

De acuerdo con la clasificación se incluyeron las Amenorreas de origen Psíquico y Neurogénico hasta donde fue posible establecer su etiología basándose exclusivamente en la determinación de Gonadotropinas Urinarias Totales y en un buen número de casos mediante el test de reserva pituitaria con Metopiriona (SU4885) resul- 
tados anteriormente descritos por nosotros (10).

Debido a las variaciones durante el ciclo de las gonadotropinas urinarias y a los valores relativos de esta prueba biológica que no nos permite tener idea de los niveles separados de FSH y $\mathrm{LH}$, creemos que la única manera probablemente de ayuda diagnóstica serían las determinaciones por separado con radio-inmuno ensayo de las gonadotropinas (FSH y LH) aún no disponibles entre nosotros; y mejor aún las determinaciones y el uso de diagnóstico de las hormonas liberadoras $\mathrm{FSH}-\mathrm{RH}$; LH - RH de acuerdo a lo propuesto por (29).

Debemos mencionar que en el intento de correlacionar la eliminación de gonadotropinas urinarias totales con la respuesta pituitaria al freno con Metopirona, estudiamos 25 casos (9) los cuales nos permitieron concluir que no existe relación alguna entre el Test de Reserva Pituitaria y los Diversos tipos de Amenorrea.

Con este grupo queremos presentar la segunda causa de Amenorrea entre nosotros en un total de 20 pacientes; la mitad de los cuales ( 11 casos) eran pacientes menores de 20 años; 7 entre 20 y 30 años y sólo 2 en la década de los 30 años.

La mayor parte de los pacientes presentaban Amenorreas Secundarias ( 17 casos) (85\%) y sólo $3(15 \%)$ Amenorrea Primaria. De ellas la mayor parte eran nulíparas ( 15 casos), y sólo 3 habían tenido embarazos previos.

En todos los pacientes con excepción de un caso con un craneofaringioma, el área y la radiografía de la Silla Turca fueron normales.

Las Gonadotropinas Urinarias fueron negativas en la mayoría de los pacientes y en sólo 2 casos se encontraron Gonadotropinas Positivas.

\section{Manejo y evolución}

En su mayoría se manejaron con terapia cíclica de estrógenos más progesterona.

Aquellas que no reanudaron sus ciclos después de la terapia indicada y sus niveles estrogénicos mostraron ser aceptables, se manejaron con clomifeno en la siguiente forma: 6 pacientes durante 36 ciclos en cursos diferentes, los cuales en un alto porcentaje presentaron ovulación (70\%) y sangradas por deprivación en un $(87 \%)$ de los ciclos (11). Solamente se obtuvo un embarazo.

De los pacientes manejados con terapia cíclica que reanudaron sus ciclos ( 14 casos) se presentaron $5 \mathrm{em}$ barazos.

No obstante el hecho de presentar seis embarazos en 20 pacientes $(30 \%)$ creemos que este grupo es el de más difícil manejo y de resultados más inciertos por la misma dificultad de su clasificación etiológica.

\section{Disgenesia gonadal}

En esta revisión como la tercera causa de Amenorrea en nuestro medio, vale la pena recordar que esta incidencia "ALTA" de disgenesia gonadal muy posiblemente está condicionada a la naturaleza de nuestra consulta especializada, a la cual son referidas las pacientes con cuadros endocrinos de importancia.

Desde que Henry Turner (36) en 1933 describió el síndrome que lleva su nombre caracterizado por: Infantilismo Sexual, Cuello en Trapecio y Cúbitus Valgus, han aparecido un sinnúmero de sinónimos. 
El advenimiento del método de la cromatina sexual en 1954, permitió a otros autores $(8,20,27,38)$, demostrar, que algunos de estos individuos a pesar de su apariencia femenina $y$ diferenciación sexual, presentaban cromatina negativa.

Morales y colaboradores (24) se refieren al síndrome denominándolo: "Disgenesia Gonadal Pura" a los casos no asociados con malformaciones y "Síndrome de Turner" propiamente dicho, a aquellos asociados a ellas.

Queremos presentar hoy los $16 \mathrm{ca}$ sos manejados en nuestra consulta de los cuales solamente uno fue de Turner verdadero, y los 15 restantes fueron clasificados como "Disgenesia Ovárica, variedad No. Turner", incluyendo un grupo de 4 hermanas con el mismo síndrome.

Los pacientes todos presentaban "Amenorrea Primaria" y las edades oscilaban entre 8 y 25 años, 12 pacientes tenían menos de 20 años y solamente 4 presentaban más de 20 años.

Todos los casos a excepción del Síndrome de Turner presentaron:

\section{A) Características Somáticas Feme- ninas. \\ B) Amenorrea Primaria. \\ C) Ausencia del Desarrollo del Se- no o Hipomastia.}

D) Genitales internos hipoplásicos.

E) Hiper-gonadotropinuria.

\section{F) Ausencia de Malformaciones}

\section{Congénitas.}

G) Cromatina Positiva.

El kariotipo de los pacientes fue de $46 \mathrm{xx}$ en 7 pacientes y 8 presentaron diversos tipos de alteraciones cro- mosómicas, caracterizadas por mosaicos, trisomías etsocromosomas, etc. alteraciones éstas descritas en estos tipos de pacientes.

Debemos llamar la atención sobre el hecho anotado por Elliot (12), quien en 1959 presentó tres hermanas con el síndrome de disgenesia gonadal, cosméticamente bien desarrolladas, que sólo presentaban osteoporosis, retardo en la edad ósea y epifisitis vertebral, todas ellas con cromatina positiva.

Queremos agregar en este escrito el fortuito hallazgo reciente de 4 hermanas con 19, 16, 14 y 8 años respectivamente, sin malformaciones, con hipoplasia gonadal, retardo óseo y ausencia de estrógenos.

\section{Manejo}

Todas las pacientes han sido sometidas, dependiendo de su eclad a terapia de suplencia para compensar su déficit estrogénico y complementar su desarrollo somático.

Los resultados han sido buenos a excepción del desarrollo mamario el cual hemos observado como muy pobre en la totalidad de las pacientes.

\section{ENFERMEDAD $Y / O$ SINDROME DE ASHERMAN}

Este Síndrome conocido como Amenorrea Traumática o Esclerosis Endometrial es debido a una atresia completa o parcial de la porción ístmicocervical del útero, del cuerpo del mismo, o de ambos.

Su historia data desde 1894 cuando fue descrita por Fritsch (13), el médico ruso Bass (5) publicó una serie de casos en 1927, pero solamente fue Asherman quien en $1948(3,4)$, analizó esta entidad como un Síndrome y las múltiples publicaciones que 
realizó a este campo le merecieron que este síndrome llevara su nombre.

Aunque el Síndrome de Asherman se describe con Amenorrea, no infrecuentemente se asocia con otras alteraciones del ciclo, y puede cursar Asintomático.

Nuestro interés en esta comunicación es presentarles a ustedes el Síndrome de Asherman como la tercera causa de Amenorrea en nuestro medio, así como el manejo y evolución de estas pacientes.

Durante la década mencionada se presentaron con Amenorrea 15 casos con Síndrome de Asherman, de los cuales 8 ocurrieron entre las edades 20 a 30 años, 6 entre las edades de 31 a 40 años y solamente uno en una paciente menor de 20 años.

La gravidez de las pacientes fue de 1 a 3 embarazos en 8 pacientes de 4 a 6 en 5 y 2 casos sin embarazo previo.

Todas las pacientes presentaban Amenorrea Secundaria, y en 2 de ellas se presentaron períodos alternos de oligo e hipomenorrea.

Las causas del Síndrome Asherman fueron: 11 pacientes con legrados post-aborto, 2 pacientes con legrado del post-parto, las 2 restantes en forma espontánea sin antecedentes de embarazo una de las cuales probablemente como consecuencia de una tuberculosis endometrial.

Como una de las pruebas en el diagnóstico de Amenorrea que realizamos de rutina es la de la "Progesterona". Esta se realizó en todas las pacientes siendo sus resultados negativos en 12 casos y positivos en 3 casos, las cuales sangraron por deprivación.

Todas las pacientes fueron sometidas a histerosalpingografías comprobatorias del diagnóstico.

\section{Tratamiento y evolución}

La pautas de manejo entre nosotros han sido en general, el manejo quirúrgico de este Síndrome, consistente en dilatación y curetaje por vía vaginal y aplicación de dispositivos intrauterinos.

En algunos casos de Sinequia ístmicc-cervical se colocaron tallos de plástico o balones intrauterinos.

Las pacientes en el post-operatorio inmediato fueron manejadas con antibióticos y terapia cíclica por 3 meses.

En 4 casos sólo se hizo tratamiento médico, debido a que las pacientes rechazaron los procedimientos quirúrgicos.

De las 11 pacientes tratadas quirúrgicamente se obtuvieron embarazos en $6(51 \%)$. De estos embarazos sólo tenemos certificación del parto en 3 casos; una paciente presentó 3 abortos consecutivos, y en 2 casos no se abtuvieron datos de la evolución; la infertilidad y aborto habitual son comunes en este grupo de pacientes.

En las 5 pacientes del grupo, intervenidas, 3 reanudaron sus ciclos, 1 se perdió del control y en otra, la Amenorrea ha persistido parece debido a una Endometritis Bacilar.

\section{Comentarios}

Nos hemos visto sorprendidos, con la alta frecuencia de este Síndrome, muchos de los cuales han ocurrido en pacientes manejadas por nosotros. El legrado del útero recientemente grávido, no deja de ser un procedimiento que implique extremo cuidado y pericia, y por ello debe ser siempre realizado o asesorado por personas con experiencia. Es seguro que el abuso o el manejo agresivo de la cureta cortante tenga mucho que ver con ello $y$ es por esto que aconsejamos el ma- 
nejo más liberal de la cureta roma. Parece que en la mayoría de los casos el embarazo precede a esta lesión traumática.

En igual forma parece que lo más aconsejable es el uso profiláctico de antibióticos en el post-legrado. También es importante destacar el hecho por el cual, sería aconsejable dejar un dispositivo intrauterino por un lapso prudencial ( 3 meses), después de un legrado traumático, particularmente después de un aborto séptico.

Referente al tratamiento creemos que lo más aconsejable es el debridamiento quirúrgico por la vía vaginal de acuerdo a lo recomendado por Louros (23), seguido de la aplicación inmediata de un dispositivo intrauterino de cualquier tipo, idealmente el tipo Lippes D: simultáneamente con uso de agentes progestacionales, los cuales contribuyen a recuperar el endometrio y evitar adherencias recurrentes.

Los resultados obtenidos por nosotros parecen así confirmarlo.

\section{NECROSIS HIPOFISIARIA (Síndrome de Sheehan)}

Es la necrosis post-parto de la $\mathrm{Hi}$ pófisis anterior dando como resultados Hipopituitarismo.

En general es causada por colapso circulatorio severo, debido a Hemorragia obstétrica y las causas son múltiples: cualquier condición que produzca Shock Severo puede desencadenar el Síndrome (30).

La patogénesis es dada por la alteración en la circulación en la Hipófisis, que puede comprometerse en 4 niveles (arterias - circulación portal capilares - venas).

La necrosis de la hipófisis anterior es producida por isquemia local debi- da a vaso-espasmo en el suministro de sangre arterial.

La incidencia se relaciona directamente con la severidad y duración del colapso circulatorio (31). Sobrevida 5-40 años. 55 mujeres por 1'000.000 de habitantes.

De acuerdo a la edad los pacientes se distribuyen así: entre los 20 y 30 años 6 casos; entre $30-40$ años, 4 casos.

El gran porcentaje de pacientes (9) eran multíparas (4) sólo 1 paciente era primigestante.

La Amenorrea fue en todas secundaria y de larga evolución 1-3 años. Al analizar cada caso se tuvo muy en cuenta la historia obstétrica acompañada de algunos informes de laboratorio como las gonadotropinas, que fueron negativas en todos los casos; sin excepción todos los pacientes presentaban un cuadro clínico de Hipoestrogenismo severo.

La Silla Turca fue normal y en sólo un caso había un hematoma subdural (Traumático).

El tratamiento en todos los casos fue la hormonoterapia de reemplazo con buena evolución de los controles obteniéndose un embarazo en una paciente de este grupo; al parecer se trataba de un Sheehan parcial.

\section{AMENORREA Y GALACTORREA (Síndrome de Chiari-Frommel)}

Este síndrome caracterizado por:

Amenorrea

Galactorrea Prolongada

Obesidad Moderada

Hipotrofia Genitales Internos y Externos (Superinvolución) 
Es el resultado de una disfunción hipotalámica de diversos orígenes que inhibe la liberación de gonadotropinas y permite la producción excesiva de hormona lactogénica por la hipófisis anterior.

Este Síndrome es raro y se presenta en mujeres jóvenes; no se presenta necesariamente en el puerperio y por lo tanto precedido de embarazo; hay pacientes que no han estado en embarazo y por lo tanto muchos piensan en un factor Psicogénico (2).

En estas condiciones tuvimos 6 pacientes; 5 de las cuales en edades entre 20-30 años y 1 entre 30-40 años.

Todas las pacientes eran multíparas. La Amenorrea por lo tanto fue siempre secundaria. La prueba de progesterona fue negativa, lo que indica ciclo ovárico en receso. La Silla Turca fue normal. El Shorr mostró hipoestrogenismo.

El tratamiento es un poco difícil. Se hizo en todos los casos con terapia cíclica con buena ovulación.

Parece prometedor el uso de citrato de clomifeno (CLOMID) para restituir los ciclos ovulatorios y aún obtener embarazos $(16,19,25)$.

\section{HIPOPITUITARISMO CON OBESIDAD DISTROFIA ADIPOSOGENITAL}

\section{S. Froehlich}

Puede ser el resultado de un trastorno funcional del área hipófisis-hipotálamo se caracteriza por obesidad y subdesarrollo genital con hipofunción hipofisiaria (28).

En este grupo estudiamos 6 pacientes 3 de las cuales tenían menos de 20 años y los otros 3 entre 20 y 30 años. Todas las pacientes tenían exceso de peso más de $80 \mathrm{kgs}$. en Amenorrea
Secundaria co ngonadotropinas bajos y negativos: Silla Turca Normal.

La prueba de progesterona fue positiva en todos los casos aunque había un hipoestrogenismo marcado.

El tratamiento se hizo con dieta de reducción de peso; terapia con progesterona sola y terapia cíclica y en un caso citrato de clomifeno (CLOMID) sin resultados.

Los ciclos artificiales pueden determinar la curación de la paciente, si se logra la reducción del peso o si la paciente se embaraza. El embarazo parece restaurar el balance endocrino en la mayor parte de las mujeres jóvenes con este tipo de hipopituitarismo; en este sentido tienen un muy importante papel la terapia gonadotrópica y los inductores de evolución.

\section{Amenorrea y alteraciones suprarrenales}

La hipofunción de la corteza adrenal ejerce efectos profundos sobre la menstruación y la fertilidad. Se pueden tener varios variantes clínicos así:

Producción excesiva corticosteroides. (S. de Cushing).

Producción excesiva de andrógenos (S. Adrenogenital).

Combinación de los anteriores.

Hiperproducción de Aldosterona (Aldosteronismo Primario).

En este grupo se estudiaron 6 pacientes de las cuales 3 eran menores de 20 años y 3 entre los 20 y 30 años; todas las pacientes nulíparas, solteras la mayoría, con Amenorrea Secundaria, Hirsutismo marcado en dos casos: hipoestrogenismo, 17 cetoesteroides bastante aumentados para nuestro medio 10 mgs. x $24 \mathrm{Hrs}$. 
Pruebas de estimulación con ACTH y supresión con Dexametosona compatibles con Síndrome adrenogenital en dos casos.

El tratamiento se hizo con esteroides principalmente prednisona en la mayoría de los casos.

"En las mujeres con hirsutismo abundante 0 en aquellas que sufren perturbaciones emocionales debido a un hirsutismo moderado debería considerarse la prueba terapéutica con corticoides. Esto es especialmente cierto cuando coexisten esterilidad y trastornos menstruales de tipo anovulatorio o por una función luteínica inadecuada, particularmente cuando las 17 cetoesteroides están en valores límites máximos de la normalidad o cuando están ligeramente aumentados. El restablecimiento de la ovulación, la mejoría de la fase luteínica del ciclo y aún el embarazo pueden presentarse a continuación del uso de un corticosteroide. El hirsutismo puede aliviarse utilizando métodos depilatorios antes de instituir la terapia con corticosteroides, el médico debe asegurarse de que no existen contraindicaciones (úlcera péptica - TBC - etc.). Así como considerar los riesgos de la supresión de la corteza Adrenal y/o sopesarlos con las necesidades terapéuticas" (17).

\section{Menopausia prematura o precoz}

"Se presenta espontáneamente antes de los 40 años, tiene lugar aproximadamente en el $8 \%$ de las mujeres. Dicho cese precoz fisiológico de la función ovárica resulta en Amenorrea en la mujer que se halla en el período de madurez sexual, interfiriendo tal vez con la reproducción. Además crea problemas en el diagnóstico diferencial y en el tratamiento la presencia de llamaradas de calor y el hallazgo de un alto nivel de gonadotropi- nas urinarias ayuda a establecer el diagnóstico. Ocasionalmente y en especial si la excreción de gonadotropinas es normal, uno debe recurrir a un sistema de medición subjetivo, la mayoría de las llamaradas ceden mediante la administración de estrógenos $(21,26)$.

En este grupo se estudiaron 5 pacientes con una edad promedio de 36 años, 2 (dos) nulíparas y 3 (tres) multíparas; en Amenorrea Secundaria, con hipoestrogenismo severo; gonadotropinas fuertemente positivas. Se les indicó tratamiento médico de suplencia con estrógenos con buena respuesta de sus síntomas principales.

\section{Amenorrea, alteraciones congénitas. Agenesia de útero; Alteraciones congénitas. Canales de Miller}

“La ausencia congénita del útero es, evidentemente causa de Amenorrea y esterilidad. Puede existir ciertos grados de duplicación en el tracto reproductor. La fusión incompleta de los conductos paramesonéfricos (Mullerianos) durante el tercer y cuarto mes de la vida fetal determina grados variables de útero y vagina bipartito. Se cree, concientemente que la etiología de la mayor parte de las malformaciones presentes en el momento del nacimiento y no determinadas por mecanismos únicos de Mutación de genes, es una combinación del medio uterino y de la predisposición genética" (39).

Con este diagnóstico se estudiaron 5 pacientes, de las cuales 3 tenían menos de 20 años y 2 por encima de los 20 .

Todas las pacientes nulíparas, en Amenorrea Primaria, con gonadotropinas positivas, cromatina sexual positiva y ginecografías que mostraban ausencia de útero y también vaginas. 
A todos los casos se les hizo tratamiento quirúrgico con reconstrucción artificial de vagina con una muy buena evolución post-operatoria.

\section{BIBLIOGRAFIA}

1 ABRAHAMS, O. L., HAWKINS, D. F. and LAWRENCE, D. M.: Estimation of ovarian size with reference to Stein-Leventhal Syndrome, Obst. and Gynec. Vol. 38, № 1, 117 124. July 1971.

2 ARGONZ, J. and DEL CASTILLO, E. B. A Syndrome characterized by estrogenic insuficiency, Calactorrhea and decrease urinary gonadotropin. J. Clin. Endocrinol. 13: 79, 1953.

3 ASHERMAN J. G.: Amenorrhea Traumática (Atrética). J. Obst. Gynec. Brit. Comm. 55: 23, 1948.

4 ASHERMAN, J.: Traumatic intrauterine adhesions. y Obstel Gynaec. Brit. Comm. 57: $892,1950$.

5 BASS, B.: Uber die verwachsurgen in der cervix uteri nach curettagen. Z. B. L. Gynaeck Si: 223. 1. 27.

6 BLACK, W. P., GOVAN, A. D. Laparoscopy and ovarian biopsy for the assessment of secundey amennorrhea. Am. J. of Obst. \& Gynec. 15, Vol. 114, № 6. p. 739, 1972.

7 BRADBURY: Proc. Soc. Exper. Biol. Am. 71 : $228,1949$.

3 BARR, M. L.: An interin note of the aplication of the Skin Biopsy test of Chromosomal sex to hermaphro-dichs. Surg. Gynec. and Obst. 99: 184, 1954.

9 COOKE, C. W., McEVOY, D., BULASCHENKO, $\mathrm{H}$. Adrenocortical and Ovarian Function in the Hirsute Women. Am. J. Obst. Gynec. Vol. 114, No 1. 65, Sept. 1972.

10 DEL CORRAL, F., SALCEDO, C. A., NIÑO, C. E. Valoración de la capacidad secretora de ACTH por la pituitaria con metopirona en pacientes con amenorrea. Rev. Col. Obst. y Ginec., Vol. 17 No 6 Nov.-Dic.

11 DEL CORRAL, F., RODRIGUEZ, A. Uso clínico del Clomifeno en anovulación. Simposio Esteroides Sexuales. Pág. 123-126, Bogotá 1968. Saadruck, Berlin 1969.

12 ELLIOT, G. A., SANDLER, A., RABINOWITZ, D. : Gonadal Disgenesin in the sisters, J.
Clin. Endocrinol. and Metabol. 19: 9961003, 1959.

13 FRITSCH, J. Citado por CARMICHAEL D. E. Asherman' Syndrome. Obst. \& Gynec. Vol. 36 No 6 p. 292-928. Dic. 1970.

14 GOETZE, D., SCHULZ, E., ROSEN, S. W. Gynecography, technique and interpretation. A. J. of Roent. Vol. 86 No 5, 866, 878, Nov. 1961

15 GOLDEMBERG, R. L., GRODIN, J. M., RODBARD, D. and ROSS, G. T. Gonadotropins in Women with amenorrhea. Am. J. of Obst. \& Gynec. Vol. 116, p. 1003, 1973.

16 GREENBLATT, R. B. "Induction of ovulation with clomifene" in ovulation. Ed. by R. B. Greenblatt Philadelphia Lippincott. 1966.

17 GREENBLATT, R. B. Cortisone in the treatment of hirsuie woman. Am. J. Obst. and Gynec. 66: 700, 1953.

18 ISRAEL, L., Trastornos menstruales y esterilidad. 5a. edición.

19 KAISER, I. H. Pregnancy following clomiphen induced ovulation in chiari frommel Syndrome. Am. J. Obst. and Gynec. 87: 334 1963.

20 KERFNOF, A. M. and STCLTE, L. A.: Two case of "Hipoplasia" of the ovaries partial primary agenesia of the gonada. Acta Endocrinol. 21: 106, 1956.

21 KEETTEL, W. C. and BRADBuRY, J. T. Premature ovarian failure, permanent and temporary. Am. J. Obst. and Gynec. 89: 83, 1964.

22 KLINEFELTER ALBRIGHT: J. Clin. End. 3 : 529, 1943.

23 LOUROS, N. C. DAREZIS y M. PONTIFIX G. : Use of intrauterine devices in the treatment of intrauterine adhesions. Fertil Steril 19: 509, 1968. No 4 July-August.

24 MORALES, A., BARREIRO, H., SANCHEZ, A. : Citogenética del Síndrome de Turner. Rev. Clínica Española № 3, 165-168, Mayo 1964.

25 PILDES, R. B. Induction of Ovulation with Clomiphene. Am. J. Obst. and Gynec. 91 : 466, 1965

26 PERLOFF, W. $H$. and SCHNEEBERG, N. C. The premature climaterium. Am. Pract. and Digest. Treat. 8: 1956, 1957.

27 POLONI, P. E., HUNTER, W. F. and LENNOX, B. Chromosomal Sex in Turrer' Syndrome 
with coaction of aorta. Lancet. 2: 1280, 1954.

28 ROGERS, J. and MITCHELL, G. W. The relation of obesit to menstrual disturbances. New Eng. J. Med. 247; 53, 1952.

29 SHALLY, A. U., MULLER E. E., ARIMURA, A. and PIZZOLATO, P. Releasings factor in human hypothalamic and neurohypophyseal extracts. J. Clin. Endocr. 27: 755, 1967.

30 SHEEHAN, H. L., DAVIS, J. Pituitary Necrosis British Medical Bulletin. 24 : 59-70, 1968.

31 SHEEHAN, H. L. The frequency of Post-Partum Hypopituitarism. The Journal of Obstetrics and Gynec. of the British Common health. 72: 103-111, 1965.

32 SHIPPEL, S.: The ovarian theca cell part IV the hipertecosis syndrome. J. Obst. Gynec. Brit. Comm. 62: 321, 1955.

33 SCHULZ, E., ROSEN, S. W. Gynecography, technique and interpretation. Am. J. of Roentg. Vol. 86 № 5, 866, 878, Nov. 1961.
34 STEIN, I. F., and LEVENTHAL, M.| L. Amenorrhea associated with bilateral polycystic ovaries. Am. J. Obst. \& Gynec. 1935, 29, 181-191.

35 STEIN, I. F. Bilateral Polycystic ovaries: significance in sterility. Am. J. Obst. \& Gynec. 50, 385-398, 1945.

36 TURRER, H. H.: Syndrome of infantilism, congenital webbed neck, and cubitus valgus Endocrinology, 23: 566, 1938.

37 WEIGEN, J. F., STEVENS, G. M. Pelvic pneumography in the diagnosis of polycystic disease of the ovary, including Stein-Leventhal Syndrome. A. J. of Roentg. Vol. 100 No 3, 680-687, July 1967.

38 WILKINS, L., GRUMACH, M. M. and VAN WYK J. J.: Chromosomal Sex in Turrer's Sindrome with coaction of aorta. Lancet. 2: $1280,1954$.

39 WILSON, D. C., and HARRIS, G. H. Congenital abnormalities of the uterus and associated malformations. J. Obst. and Gynec. Brit. Comm. 68: 841, 1961. 